\title{
Visual working memory for line orientations and face identities
}

\author{
YUHONG V. JiANG \\ University of Minnesota, Minneapolis, Minnesota \\ Won MoK SHIM \\ Massachusetts Institute of Technology, Cambridge, Massachusetts \\ AND \\ Tal Makovski \\ University of Minnesota, Minneapolis, Minnesota
}

\begin{abstract}
Previous studies have shown that the number of objects we can actively hold in visual working memory is smaller for more complex objects. However, complex objects are not just more complex but are often more similar to other complex objects used as test probes. To separate effects of complexity from effects of similarity, we measured visual memory following a 1-sec delay for complex and simple objects at several levels of memory-to-test similarity. When memory load was one object, memory accuracy for a face (a complex attribute) was similar to a line orientation (a simple attribute) when the face changed in steps of $10 \%$ along a morphing continuum and the line changed in steps of $5^{\circ}$ in orientation. Performance declined with increasing memory load and increasing memory-to-test similarity. Remarkably, when memory load was three or four objects, face memory was better than orientation memory at similar changed steps. These results held when memory for line orientations was compared with that for inverted faces. We conclude that complex objects do not always exhaust visual memory more quickly than simple objects do.
\end{abstract}

Whether it is crossing a busy street, playing team sports, or driving, many daily activities require the buffering of visual information for a short time after its disappearance (Baddeley, 1986; Hollingworth, Richard, \& Luck, 2008; Logie, 1995). The amount of information actively held in visual short-term memory is severely limited. This limitation is often demonstrated by showing observers an array of visual objects, taking them away, and probing memory with a changed test display after a short delay (Baddeley, 1986; Hollingworth et al., 2008; Logie, 1995; Pashler, 1988; Phillips, 1974). Using this procedure, most visual short-term memory studies have put the upper capacity limit at three or four objects (Alvarez \& Cavanagh, 2004; Awh, Barton, \& Vogel, 2007; Irwin \& Andrews, 1996; Luck \& Vogel, 1997).

Extensive research has been devoted to characterizing factors that influence the capacity of visual short-term memory. This research shows that, much like verbal shortterm memory, visual short-term memory is limited by the number of "chunks," where each chunk is a single visual object (Luck \& Vogel, 1997). However, researchers continue to debate whether the number of available chunks is fixed for all kinds of visual stimuli, or whether it varies depending on the complexity of visual features (Alvarez \& Cavanagh, 2004; Awh et al., 2007; Eng, Chen, \& Jiang,
2005; Makovski \& Jiang, 2008a; Zhang \& Luck, 2008). Much progress has been made in addressing this question, but no clear consensus has been reached.

In order to test the relationship between memory capacity and object complexity, we must first clarify how "complexity" is measured. Various definitions have been proposed, including "figure goodness," indexed by a set of rotation and reflection transformations (Garner, 1962; Garner \& Sutliff, 1974); "perimetric complexity," indexed by the ratio between the square of perimeter and "ink" area of a shape (Attneave \& Arnoult, 1956; Pelli, Burns, Farell, \& Moore-Page, 2006); and "informational load," indexed by visual search slope (Alvarez \& Cavanagh, 2004; Eng et al., 2005). The index used most often in studies of visual short-term memory is informational load, measured by the slope relating visual search speed to the number of search objects. Complex objects take longer to process, leading to a steeper search slope in comparison with simple objects. The index of informational load corresponds well with figure goodness (Makovski \& Jiang, 2008a), which corresponds well with perimetric complexity (Attneave, 1957; Garner \& Sutliff, 1974).

Relying on informational load, Alvarez and Cavanagh (2004) proposed that the number of available "slots" in visual short-term memory is inversely related to the in-

Y.V. Jiang, jiang166@umn.edu 
formational load carried by the objects. Complex objects such as random polygons, cubes with sides of variable shading, and unfamiliar faces carry heavier informational load and fill up visual working memory more quickly than simple objects such as color patches and line orientations (Alvarez \& Cavanagh, 2004; Eng et al., 2005). These findings support the flexible-slot hypothesis, according to which visual working memory has fewer slots for objects with heavier informational load.

Advocates for a fixed-slot hypothesis pointed out that visual working memory may contain a fixed number of slots, but multiple slots are needed to hold a complex object (Luck \& Zhang, 2004). Furthermore, a better ability to detect changes in simple objects does not necessarily mean that simpler objects are better retained in visual short-term memory. Instead, because simple objects are often highly distinguishable from other simple objects used as memory probes, the change from one simple object to another produces a larger change signal than that from one complex object to another. Because larger changes are easier to detect (Zhou, Kahana, \& Sekuler, 2004), the complexity effect observed previously may reflect a similarity effect. To find out whether complexity influences memory representation, experimenters must equate the size of change for simple and complex objects.

Attempts to dissociate similarity and complexity have been made previously. Awh et al. (2007) found that change detection was simple when a memorized cube changed into a Chinese character or vice versa, but the task became more difficult when the change was within category. These researchers suggested that memory for complex objects can be as accurate as memory for simple objects, given that a large change was used to probe memory. However, when the change was made between categories, detection performance may not have been supported by memory of the exact shape (a complex attribute), but by memory of salient features that distinguish the two categories (a simple attribute). To ensure that memory for simple and complex objects is being investigated, a different approach must be taken. In this study, we introduced such an approach, in which only within-category changes were made, but the size of the change from memory to test was comparable between simple and complex objects. This design allowed us to address the complexity effects in a short-term memory delay task.

To examine whether visual memory in a short-term task is exhausted more quickly by more complex objects, we parametrically manipulated the similarity between a memory object and a test probe for two types of objects: line orientations and human faces. The parametric manipulation of memory-to-test similarity allowed us to choose psychologically equivalent changes in the face task and the line orientation task at a memory load of 1 . For example, at load 1, performance may be comparable when faces change in $M \%$ along a face morph and the lines change in $N$ degrees in orientation; accordingly, we tested whether these changed steps still produced comparable levels of performance for faces and line orientations at higher memory load.

\section{EXPERIMENT 1 Faces and Line Orientations}

We tested two types of stimuli that differed significantly in subjective complexity: face identity and line orientation. Figure 1 shows the full set of stimuli used in this experiment.

At the 2007 Psychonomic Society meeting, an audience of about 100 psychologists were shown one line and one face (Figure 1) among other objects, presented one at a time. All but 2 psychologists rated the face as more complex than the line. The psychologists' intuition was verified in laboratory testing, where 14 participants (age range, 19 to 30 years) rated the subjective complexity of several objects, including a face and a line shown in Figure 1. On a 7-point scale, with 1 being very simple and 7 being very

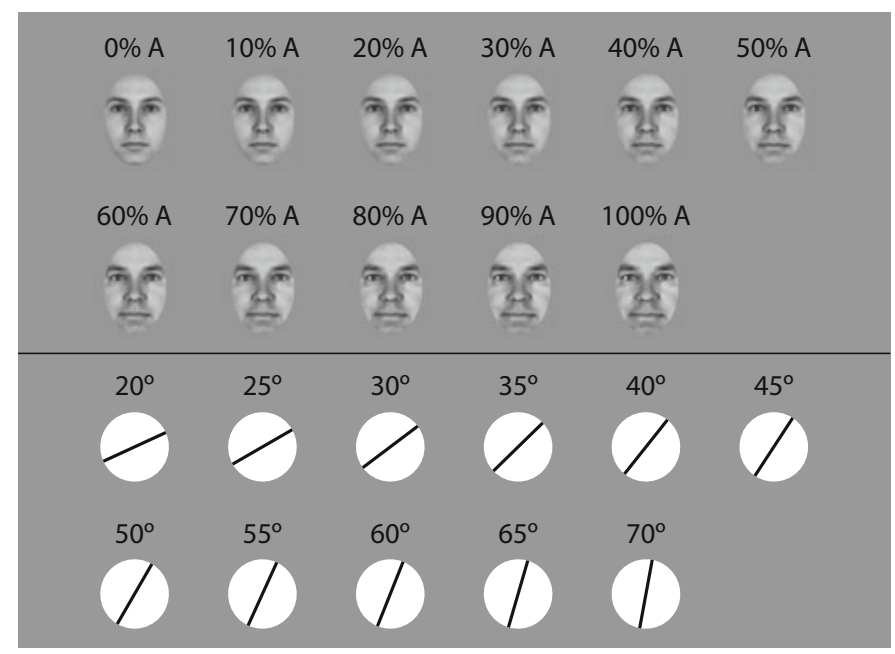

Figure 1. Eleven faces and line orientations used in Experiment 1. 
complex, the average rating was $5.6(S E=0.4)$ for the face and $2.1(S E=0.2)$ for the line. Every rater gave a higher complexity rating to the face than to the line $(p<.001)$. The subjective rating results fit well with neurophysiological processing hierarchy, where line orientations are processed early by $\mathrm{V} 1$ neurons, whereas face identities are coded later by cells in the inferior temporal cortex (GrillSpector \& Malach, 2004).

A second reason to test face identity and line orientation is that, unlike shaded cubes or Chinese characters that can only be sampled from a finite set, faces and line orientations can be changed parametrically. This property enables us to sample various levels of memory-to-test similarity, providing a psychometric function of memory performance at different levels of memory-to-test similarity (Zhou et al., 2004). We then measure performance at progressively higher memory load. If visual short-term memory for complex objects is loaded up more quickly by more complex objects, increasing memory load should impair performance more in the face task than in the line task.

\section{Method}

Participants. Five observers (age range, 27 to 32 years), including 2 authors (Y.V.J. and W.M.S.) and 3 naive participants, were tested individually in a room with normal interior lighting. They sat approximately $57 \mathrm{~cm}$ from a 19 -in. monitor. Experiments were programmed with Psychophysics Toolbox (Brainard, 1997; Pelli, 1997) implemented in MATLAB.

Stimuli. Two types of stimuli were used in this experiment: upright faces and line orientations (Figure 1).

Faces. Eleven faces were selected from the Max Planck face database (Troje \& Bülthoff, 1996) (faces.kyb.tuebingen.mpg.de/). All faces were converted to grayscale and preprocessed to have equal overall luminance and contrast. Each face subtended $4.88^{\circ} \times 6.05^{\circ}$. At one extreme was a face-Face A-that contained $100 \%$ of an individual's face. At the other extreme was an average face with $0 \%$ of Face A (Blanz \& Vetter, 2003). The 11 faces were morphs of Face A onto the average face in steps of $10 \%$ morph. Thus, the faces contained $100 \%, 90 \%, 80 \%$, and so on, down to $0 \%$ of Face A (Figure 1 , top). The middle 9 faces were used as the memory stimuli. The changed test face could differ from the memory face by $10 \%, 20 \%$, $30 \%, 40 \%$, or $50 \%$ toward either Face A or the average face.

Lines. We placed a black line against a white disk to form the line stimulus (line length, $4.88^{\circ}$; line width, $0.16^{\circ}$ ). The line was placed against a disk to reduce interaction among multiple lines that tend to form emergent configural contours (Alvarez \& Cavanagh, 2008; Delvenne \& Bruyer, 2006; Jiang, Chun, \& Olson, 2004). The 11 lines ranged from $20^{\circ}$ to $70^{\circ}$ in 11 increasing steps of $5^{\circ}$ (Figure 1 , bottom). The middle nine orientations were used as the memory stimuli. The changed test line could differ from the memory line by $5^{\circ}, 10^{\circ}, 15^{\circ}, 20^{\circ}$, or $25^{\circ}$, clockwise or counterclockwise.

Task. Figure 2 illustrates the basic trial sequence. On each trial, one to four objects from a given category (lines or faces) were presented on the memory display against a gray background. Objects on the memory display were sampled without replacement from the middle nine examples equally often. Memory objects were presented equidistantly from one another, with each object's center being $4.68^{\circ}$ away from fixation. To ensure adequate encoding, the duration of the memory display was proportional to memory load: $500 \mathrm{msec}$ for load 1, 1,000 msec for load 2, 1,500 msec for load 3, and 2,000 msec for load 4 . The presentation duration was within the typical range of encoding duration used in visual short-term memory studies (Chen, Eng, \& Jiang, 2006; Eng et al., 2005; Makovski, Shim, \& Jiang, 2006) and was the same for line orientations and faces. Participants were allowed to move their eyes. Following the presentation of the memory display, a blank retention interval (with a gray screen) lasted $1,000 \mathrm{msec}$, after which two horizontally arrayed test probes were presented. The test probes were centered on one of the memory objects. Participants were told to compare the memory object at the probed location with the two test probes. One test probe was identical to the memory object at that location and the other was changed by one of five steps. Each step in the line orientation task was $5^{\circ}$, and each step in the face task was $10 \%$ morph. The direction of the change was balanced overall, but if the memory object was toward an extreme, the changed item was typically less extreme. For example, if the memory object were a $90 \%$ Face A and the change were one step, the changed probe could be either the $80 \%$ Face A or the $100 \%$ Face A, equally often. But if the change were two steps, the changed item could only be the $70 \%$ face. ${ }^{1}$ The same rule of stimulus sampling applied to line orientations.

Participants pressed the left or right key to indicate whether the left or right test probe matched their memory at the probed location. A beep was presented after each incorrect response. To minimize verbal recoding, the task was carried out with concurrent articulatory suppression (Baddeley, 1986). Participants repeated a three-letter word, specified before each block, as rapidly as they could, throughout each trial.

Design. We employed a full factorial design of two stimulus types (faces or line orientations), four memory loads $(1,2,3$, or 4$)$, and five sizes of change from memory to testing (one to five steps). Trials from all conditions were randomly intermixed in a given testing session of 360 trials (divided into eight blocks). One author and 1 naive participant completed six sessions; the other participants completed four sessions.

\section{Results}

Figure 3A shows memory accuracy for one face or one line at five changed steps. Accuracy was lower when the similarity between the memory item and the changed

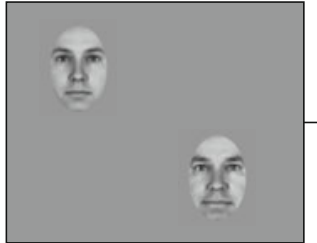

Encoding: $0.5 \mathrm{sec} \times$ Load

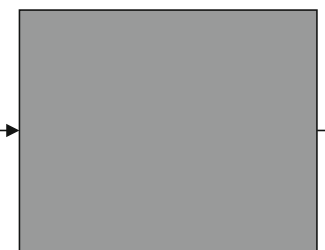

Retention: $1 \mathrm{sec}$

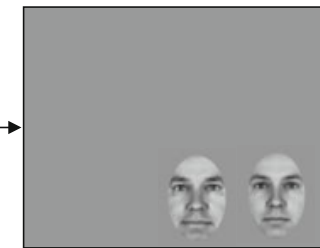

Probe: Which Matches?

Figure 2. Trial sequence used in Experiment 1. Observers remembered one to four objects from a single category (lines or faces) and reported whether the left or right test stimulus matched the memory object previously centered at the probe location. One of the test stimuli was the same as the memory object at that location; the other was changed in one of five steps. 
probe was greater $[F(4,16)=41.20, p<.001]$. This result confirmed previous findings (Awh et al., 2007; Zhou et al., 2004). Face task accuracy was similar to line task accuracy $(F<1)$. The interaction between stimulus type (faces vs. line orientations) and memory-to-test similarity (Steps 1-5) was insignificant with Greenhouse-Geisser correction for sphericity $[F(1.7,6.8)=3.59, p>.09]$. The borderline significance was driven by numerically better performance in the line task than in the face task at Step 2, but similar performance in the two tasks at other steps. Individual $t$ tests failed to show significant differences between line and face memory at any of the steps tested $(p \mathrm{~s}>.15)$. Thus, the five changed steps (in units of $5^{\circ}$ for line orientation and $10 \%$ for face morph) produced similar levels of accuracy in the face memory and line memory tasks, except that the line performance was numerically better than face performance at Step 2 .

Figures $3 \mathrm{~B}$ and $3 \mathrm{C}$ show performance at various memory loads for lines and faces. For both types of stimuli, the psychometric curve shifted downward and rightward as memory load increased $[F(3,12)=7.52, p<.004$ for faces; $F(3,12)=50.72, p<.001$ for lines]. In order for performance to reach the same level, a greater change was needed as memory load increased. The shift occurred numerically for all memory loads, resulting in a significant linear trend of load $[F(1,4)=10.80, p<.03$ for faces; $F(1,4)=211.15, p<.001$ for lines]. There was a significant reduction in performance from load 1 to load 2 for lines $[F(1,4)=253.99, p<.001]$ and a marginal reduction from load 1 to load 2 for faces $[F(1,4)=5.71$, $p<.08]$.

Was the memory load effect greater for more complex stimuli? The answer, judging by our data, was "no." Increasing memory load led to a greater decline in the line orientation performance than in the face performance. Face performance was superior to line performance at load $3[F(1,4)=41.12, p<.003]$ and load $4[F(1,4)=$ $7.57, p<.05]$. Driven by a smaller load effect in the face task, the interaction between stimulus type (faces or line orientations) and memory load was significant $[F(3,12)=$ $4.81, p<.02]$. The advantage of faces over lines was eliminated at the smallest change step, possibly due to a floor effect $[F(4,16)=7.66, p<.001]$, for the interaction between stimulus type and size of change.

\section{Discussion}

Experiment 1 reveals two noteworthy findings. First, in both the line and the face memory tasks, overall accuracy declined as memory load increased. At higher memory load, the test probe must be more different from the memory object for performance to reach a certain threshold-say, 75\% (Palmer, 1990). The reduction in memory accuracy is significant, even when load increased from one to two objects, both of which are within the typically assumed memory capacity (Cowan, 2001). These results indicate that memory precision can still improve within the conventional memory capacity of about three or four. This improvement may be attributed to the redundant representations of a single memory object in multiple available slots (Zhang \& Luck, 2008). Second, the effect of memory load on performance was not greater in the face task than in the line task; the opposite was found. There was no evidence that more complex objects load up visual memory more quickly in a visual short-term memory task. The idea that visual short-term memory capacity is always inversely related to object complexity is unsubstantiated.

\section{EXPERIMENT 2 \\ Line Orientations and Inverted Faces}

This experiment aimed to replicate Experiment 1 and to address a frequent criticism of that experiment. Many researchers have argued that the perception of upright faces relies on a special "face module" (Duchaine \& Nakayama, 2005; Moscovitch, Winocur, \& Behrmann, 1997). Perhaps visual memory for faces is unique because it is enhanced by the specialized face system (Curby \& Gauthier, 2007). To evaluate this possibility, we tested participants in a sec-
A

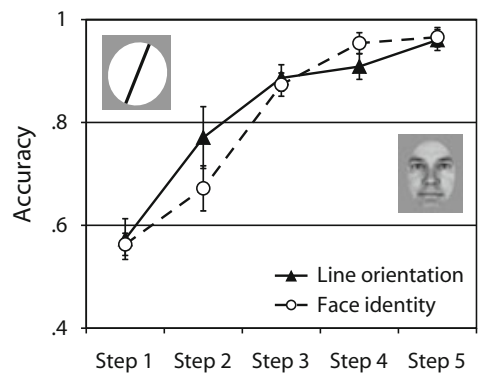

Size of Changed Signal Between Memory and Testing Stimuli (Each Step $=5^{\circ}$ for Lines and $10 \%$ for Faces)

\section{B}

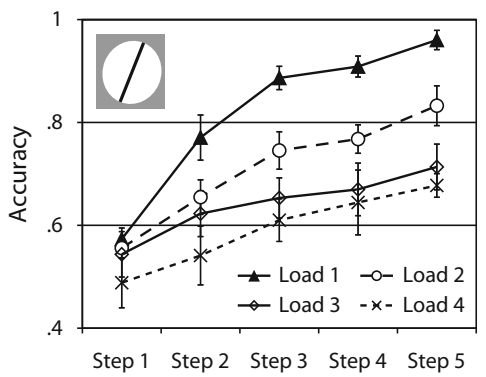

Size of Changed Signal

Between Memory and Testing

Stimuli (Each Step $=5^{\circ}$ )

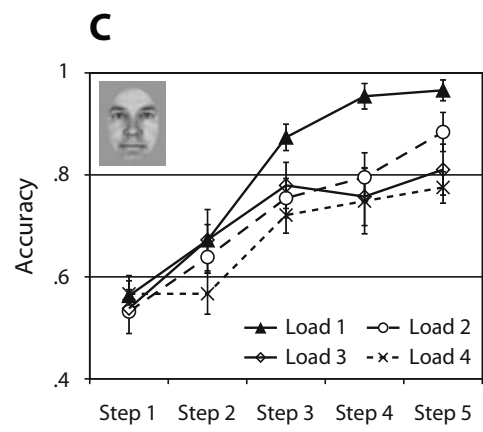

Size of Changed Signal Between Memory and Testing Stimuli (Each Step $=10 \%)$

Figure 3. Results from Experiment 1. (A) Accuracy for one line or one face at five changed steps. (B) Accuracy for one to four lines. (C) Accuracy for one to four faces. Error bars show \pm 1 standard error of the mean across participants. 
A

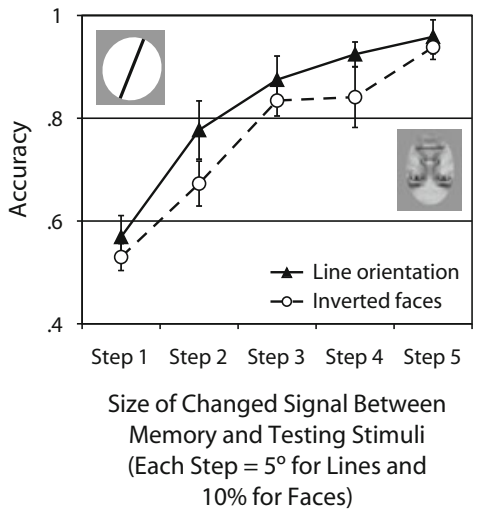

B

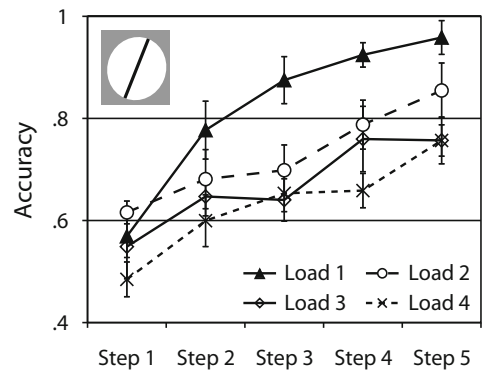

Size of Changed Signal

Between Memory and Testing

Stimuli $\left(\right.$ Each Step $=5^{\circ}$ )

\section{C}

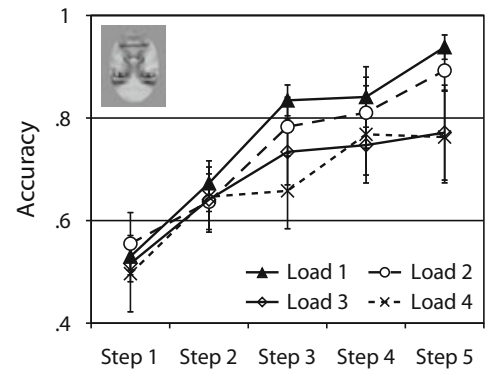

Size of Changed Signal

Between Memory and Testing

Stimuli (Each Step = 10\% )

Figure 4. Results from Experiment 2. (A) Memory accuracy for one line was better than for one inverted face. (B) Memory accuracy for one to four lines. (C) Memory accuracy for one to four inverted faces. Error bars show \pm 1 standard error of the mean across participants.

ond experiment, where line orientations were compared with inverted faces. Studies on both normal adults (Young, Hellawell, \& Hay, 1987) and brain-damaged patients with object agnosia or prosopagnosia (Farah, Wilson, Drain, \& Tanaka, 1995; Moscovitch et al., 1997) have shown that inverted faces are handled not by the specialized face system but by the general object processing system used for nonface objects. If the greater memory load effect on line memory than on face memory is restricted to memory for upright faces, it should not generalize to memory for inverted faces.

\section{Method}

We tested participants on visual working memory tasks, using the same line and face stimuli as in Experiment 1, except that the faces were turned upside-down. Four of the 5 participants from Experiment 1 completed this experiment. Other than the inversion of faces, other aspects of the experiment were the same as those of Experiment 1.

\section{Results}

As in Experiment 1, increasing memory load was more detrimental to line memory than to face memory, even though the faces were inverted (Figure 4). This difference was reflected by a significant interaction between stimulus type (lines or inverted faces) and memory load $[F(3,9)=$ $4.76, p<.03]$. At a memory load of 1 , line performance was significantly better than inverted-face performance $[F(1,3)=11.73, p<.04]$. This difference was eliminated at higher memory loads $(F \mathrm{~s}<1)$. Indeed, at load 4 , performance was numerically better in the inverted-face task than in the line task.

Besides their different sensitivity to increased memory load, face and line memory share some similar features. Memory accuracy declined when load increased $[F(3,9)=$ $21.17, p<.001]$ and when the memory-to-test similarity increased $[F(4,12)=33.39, p<.001]$. The sensitivity to increased memory-to-test similarity was comparable for face and line tasks $(F<1$ in the interaction between memory-to-test similarity and stimulus type, and $F<1$ in the three-way interaction between load, stimulus type, and memory-to-test similarity).

\section{Discussion}

Experiment 2 replicated the results of Experiment 1. Again, memory accuracy declined with increasing load for both line orientations and inverted faces. This decline was less dramatic for inverted faces than for line orientations, even though the former was more complex.

One notable difference between Experiments 1 and 2 was that - although performance was comparable for upright faces and line orientations at load 1 -it was better for line orientations than for inverted faces. This difference reveals a face-inversion effect, where perception and memory of upright faces are superior to those of inverted faces (Farah, Tanaka, \& Drain, 1995; Yin, 1969).

\section{EXPERIMENT 3 Informational Load}

A skeptical reader might dismiss the former results by arguing that faces may not be more complex than line orientations. After all, subjective rating is just that-subjective. Here, we provide an objective index of complexity by measuring the informational load of faces and line orientations. Informational load was first introduced by Alvarez and Cavanagh (2004), who measured object complexity by obtaining the slope of the linear function relating search response time (RT) to display set size in a visual search task (Wolfe, 1998). This measure is appealing as an alternative to subjective rating. However, search slope as a measure of informational load is also controversial, because it confounds an object's intrinsic complexity with its relative similarity to other objects (Duncan \& Humphreys, 1989). Nonetheless, informational load has been an influential concept in studies of visual working memory, so we measure it in Experiment 3 to provide converging evidence for the idea that faces are more complex than lines. 
We tested two versions of the visual search experiment that differed in the homogeneity of the distractor stimuli, which was known to affect search efficiency (Moraglia, 1989; Wolfe, Friedman-Hill, Stewart, \& O'Connell, 1992). In Experiment 3A, participants searched for a target among homogeneous distractors that differed from the target by three or five steps. In Experiment 3B, participants searched for a target among heterogeneous distractors that differed from the target by \pm 3 and \pm 5 steps. We intended to find converging evidence from Experiments $3 \mathrm{~A}$ and $3 \mathrm{~B}$ for the idea that faces carry heavier informational load than lines do.

\section{Experiment 3A}

\section{Method}

Participants. The same individuals who took part in Experiment 1 took part in this experiment.

Design and Procedure. Each participant completed a visual search task, where they searched for an odd item among two, five, or eight identical distractors (Figure 5). The target was present on every trial. The distractors were selected from the same category (faces or lines) as the target but differed from the target in three or five steps (see Experiment 1's use of "steps"). All items were presented in randomly selected locations sampled from a $4 \times 4$ imaginary grid $\left(16.88^{\circ} \times\right.$ $\left.16.88^{\circ}\right)$. Items were slightly jittered with respect to the center of a cell, to reduce colinearity. Following Alvarez and Cavanagh (2004), the search display was preceded by the presentation of a target stimulus for $500 \mathrm{msec}$. Participants searched for the cued target, which was also the odd (unique) item on the search display, and pressed the space bar upon detection. RT was defined as the interval between search display onset and the space bar response. The display was then replaced by an array of digits ranging from 1 to 9 . Participants typed in the digit occupying the target's location; this response yielded an accuracy measure. Each participant completed 432 trials, divided randomly and evenly into two stimulus types (faces or lines), three set sizes (three, six, or nine total items), and two target-to-distractor similarity levels (three or five steps of difference between the target and distractors).

\section{Results}

Figure 6A shows visual search RT on correct trials. The search slope on face trials was $285 \mathrm{msec} /$ item when the target and distractor faces differed by $30 \%$ morph, and $134 \mathrm{msec} /$ item when they differed by $50 \%$ morph. The search slope on line trials was $25 \mathrm{msec} /$ item when the target and distractor lines differed by $15^{\circ}$, and $-5 \mathrm{msec} /$ item when they differed by $25^{\circ}$. The interaction between set size and stimulus type was significant $[F(2,8)=25.94, p<$ $.001]$, confirming that search slopes were steeper for faces than for lines. Error rates mirrored RT data (Figure 6B). The search results suggest that faces carry heavier informational load than lines do.

\section{Experiment 3B}

\section{Method}

Participants. Four participants completed Experiment 3B. They were previously tested in other experiments reported here.

Design. This experiment was similar to Experiment 3A except for the following changes. The critical difference is that distractors on the search display were heterogeneous. Specifically, on each trial, participants were presented with a cue object at fixation along with three, five, or seven search items placed equidistantly on an imaginary circle (radius $=7.5^{\circ}$ ). One of the search objects was the same as the cue object at fixation and the others were distractors. The distractors were selected randomly with replacement from items that were \pm 3 and \pm 5 steps away from the target. The target was defined by the central cue (rather than by a precued object), because it was no longer an odd item. The presence of the central cue also ensured that search errors did not result from a failure to remember the identity of the cue. Participants pressed the space bar upon target detection and typed in the digit occupying the target's location. Each participant completed 432 trials, divided randomly and evenly into two stimulus types (lines or faces), three set sizes (three, five, or seven), and nine possible targets.

\section{Results}

Figure 7 shows results from Experiment 3B. Search accuracy was above $90 \%$ and was not significantly affected by task $[F(1,3)=2.92, p>.15]$, set size $[F(2,6)=2.60$, $p>.15]$, or their interaction $(F<1)$. Mean RT on correct trials was significantly longer in the face task in comparison with the line task $[F(1,3)=55.68, p<.005]$. In both tasks, RT was longer when there were more items on the display $[F(2,6)=54.80, p<.001]$. However, a significant interaction between task and set size showed that search slope was steeper in the face task $[F(2,6)=6.88, p<$ $.028]$. The slope was $265 \mathrm{msec} /$ item in the line task and $374 \mathrm{msec} /$ item in the face task.

\section{Discussion}

As a control experiment, Experiment 3 demonstrated that faces carry heavier informational load than line orientations do. Search slopes were steeper in the face search task than in the line orientation search task, both when the distractors were homogeneous and when they were heterogeneous. By this measure, lines carry lower informational load than do faces and can be considered a simpler type

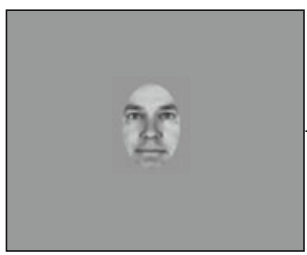

Target Cue: $0.5 \mathrm{sec}$

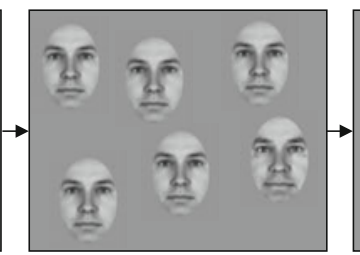

Search Array (Until Response)

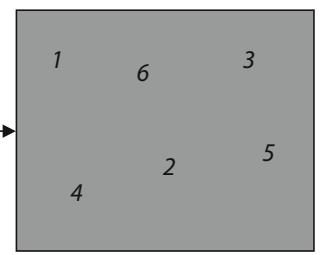

Which Digit Occupied the Target Location?

Figure 5. Trial sequence used in Experiment 3A. Observers searched for the cued target. Upon target detection, they pressed the space bar and typed in the digit behind the target item. Search set size was three, six, or nine. 
A Visual Search (Target and Distractors Differ by Three Steps)

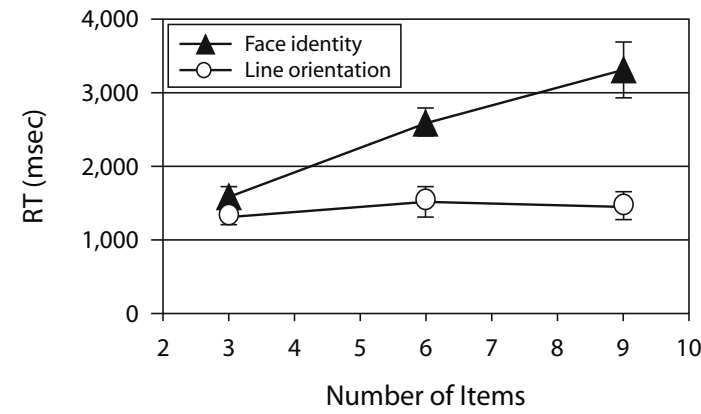

B Visual Search (Target and Distractors Differ by Three Steps)

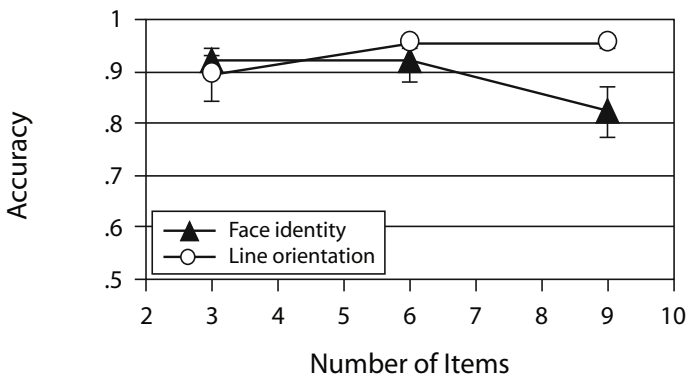

Visual Search (Target and Distractors Differ by Five Steps)

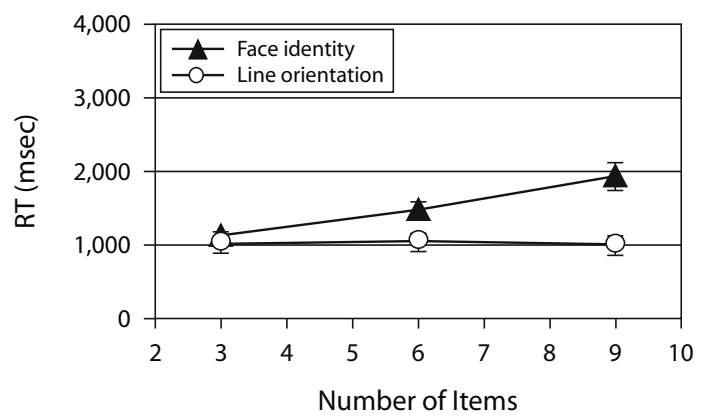

Visual Search (Target and Distractors Differ by Five Steps)

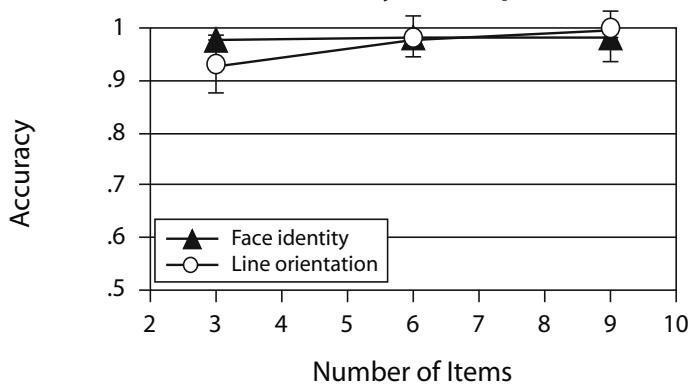

Figure 6. Results from Experiment 3A. (A) Visual search response time (RT). (B) Accuracy. The distractors were homogeneous. Error bars show \pm 1 standard error of the mean across participants.

of stimulus. We recognize, however, that search slope is a controversial concept as a measure of object complexity (Awh et al., 2007; Duncan \& Humphreys, 1989). In fact, the measured informational load for a given type of stimulus changed significantly depending on the nature of the distractors. Search slopes were steeper when the distractors were heterogeneous rather than homogeneous (Moraglia, 1989; Wolfe et al., 1992). Thus, search slope (or "informational load") cannot be taken as a straightforward index of an object's "intrinsic" complexity. None- theless, our results indicate that search efficiency can be dissociated from working memory load. A stimulus that produces more efficient search (e.g., the line orientations) might fill up working memory more quickly than would another stimulus (e.g., the faces).

\section{GENERAL DISCUSSION}

This study presents one of the first comprehensive data sets on visual memory for two types of stimuli differing
A

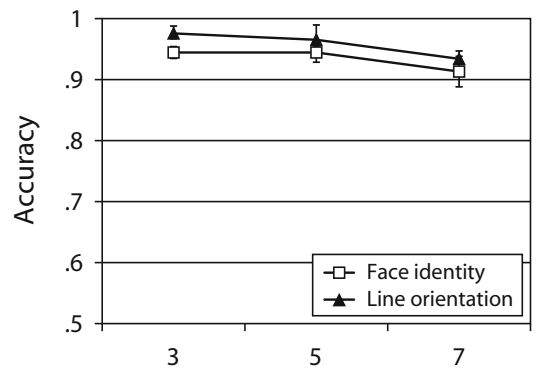

B

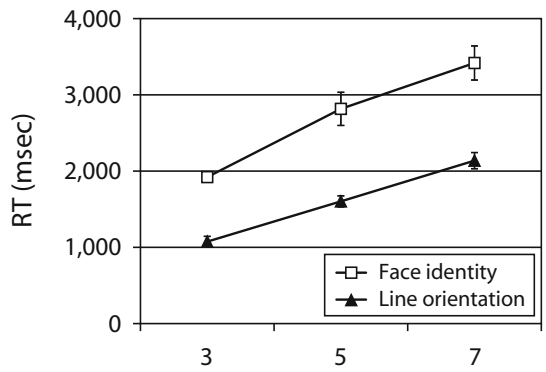

Figure 7. Results from Experiment 3B. (A) Visual search accuracy. (B) Response time (RT). The distractors were heterogeneous. Error bars show \pm 1 standard error of the mean across participants. 
significantly in complexity. By systematically varying the similarity between a memory object and a test probe, we measured performance in a short-term memory task for line orientations and faces at different memory loads. We observed the following results.

First, increasing memory load impaired memory for line orientations more than memory for faces. At a low memory load of 1 , face memory was equal to or worse than line orientation memory when faces changed in steps of $10 \%$ in face morph and lines changed in steps of $5^{\circ}$ in orientation. At a high memory load of 3 or 4 , face memory was better than line orientation memory at these steps. These results were unexpected because faces are intuitively more complex, an intuition supported by the visual search results. Our study provides evidence against the idea that complex objects always fill up active visual memory more quickly than simple objects do. Similar results were obtained when comparing memory for inverted faces and line orientations.

Why did line orientations more quickly load up visual memory than faces in our study, whereas the opposite might be expected from previous findings? Previous visual short-term memory studies that employed large memoryto-probe changes have shown that memory capacity for faces is between one and two (Curby \& Gauthier, 2007; Eng et al., 2005) and for line orientations is just under four (Jiang et al., 2004; Luck \& Vogel, 1997). There are several reasons why our results are different. First, previous studies have not controlled for the memory-to-probe similarity between lines and faces. These studies typically used lines that differed by at least $45^{\circ}$ from memory to testing, a difference that may be psychologically greater than that produced by changing one face to another. Second, by using large changes, previous studies may have introduced global configural changes that specifically aided memory for line orientations (Alvarez \& Cavanagh, 2008; Delvenne \& Bruyer, 2006; Jiang et al., 2004; Luck \& Vogel, 1997). This kind of configural memory could be of limited use when the orientation change is small, as in our study. When the similarity between memory and test stimuli was controlled for, face memory was more robust at high load, perhaps because multidimensional stimuli (e.g., faces) do not interact with each other as much as single-dimensional stimuli (e.g., lines) do (Rouder, 2001).

The second finding from our results is that in both line and face memory tasks, performance was worse when the test probe was more similar to the memory object. This finding shows that visual memory has limited resolution, even when the memory delay is short (Palmer, 1990; Zhang \& Luck, 2008; Zhou et al., 2004). In addition, the greater the memory load, the poorer the memory precision averaged across all memory objects; that is, a larger change must be made from the memory object to the test probe for change detection to reach the same threshold performance. The reduction in averaged memory precision was seen even when the load increased from 1 to 2 , both of which were within the conventionally accepted memory capacity.

What accounts for the reduction in memory precision averaged across all items? At least two hypotheses have been proposed. According to the "flexible resolution" view (Wilken \& Ma, 2004), the greater the memory load, the greater the number of items retained in memory. However, each item was retained with poorer memory resolution. According to the "fixed-slot, fixed-resolution" view, a fixed number of objects (e.g., 3 out of $N$ items) were held in visual working memory, each with a fixed resolution. When the memory load exceeded the number of available slots, some items would not be retained in memory, so the resolution to those items would be infinitely low. The measured memory precision on any trial was the average between those retained with (fixed) high resolution and those not retained in memory (Zhang \& Luck, 2008). Recent results combining psychophysical data with modeling have supported the latter view (Rouder et al., 2008; Zhang \& Luck, 2008). By measuring the kinds of errors people commit in a color working memory task, Zhang and Luck were able to show that the errors were well modeled as the sum of high-precision memory for three colors and random guesses for the others. When the color memory load was 1 , the precision of memory response was better than that at load 3. These data were also modeled by assuming that the three available slots were each used to hold the single color, producing redundant gain (Rouder et al., 2008; Zhang \& Luck, 2008).

In spite of a few caveats (see the Appendix), our experimental paradigm allowed crude modeling of the data. Specifically, we modeled the error distribution (the proportion of trials on which participants reported a changed test stimulus as the same as the memory) at different memoryto-test similarity levels using a generalized error distribution. ${ }^{2}$ The spread of this distribution became broader as memory load increased from 1 to 4 in the line orientation task, with no clear evidence for an asymptote (see the Appendix). Although this result may be taken as evidence for the "flexible resolution" view (which predicts a widening of error distribution at high memory load), it is also consistent with the "fixed-resolution" view, as long as one assumes that the lines received redundant representations in four or more available slots (Zhang \& Luck, 2008). In the face memory task, the error distribution became broader from one to two faces and did not change from two to four faces. These results may also be taken as evidence for the "fixed-resolution" hypothesis as long as one assumes that a single face received redundant representations in two (but not more) slots. Future studies that vary memory load at a wider range are needed to evaluate these assumptions (Zhang \& Luck, 2008).

Although we have only tested visual memory for faces and line orientations, the approach taken here can be used to test other types of stimuli, such as color, luminance, and complex shapes that continuously deform. One could, for instance, match memory performance on colors and complex shapes at load $1,{ }^{3}$ and probe performance for colors and shapes at greater memory loads. This work is necessary in order to test the generality of the conclusions reached here. A promising approach is to create a new set of stimuli where the simple and complex objects are products of the same morphing algorithm. This approach is needed because faces and line orientations differ in so 
many dimensions that a direct comparison can only be made crudely.

How much of what is measured in a short-term delayed memory task reflects processes specific to visual shortterm memory, as opposed to visual long-term memory? ${ }^{4}$ Unfortunately, visual short-term memory is not the only system that can contribute to performance in a short-term delayed task. Visual long-term memory will always contribute to an ongoing task (Makovski \& Jiang, 2008b), even when the task involves perception rather than memory (Chun \& Jiang, 1998) and when the memory delay is short (Buttle \& Raymond, 2003). The contamination from visual long-term memory to a short-term task is an issue in all existing short-term memory studies. In our study, we have tried to minimize the differential impact of long-term memory on faces and line orientations by using unfamiliar faces (Buttle \& Raymond, 2003) and inverted faces (Jackson \& Raymond, 2008). Given that familiarity effects in face working memory tasks were restricted to faces with super familiarity (e.g., faces of celebrities or friends) and were eliminated with face inversion (Eng, Chen, \& Jiang, 2006; Jackson \& Raymond, 2008), we are confident that our results are not products of long-term familiarity effects. Nonetheless, future studies should further address how familiarity (and long-term memory) modulates working memory, and whether any modulation is due to an interaction between familiarity and subjective complexity.

To summarize, our study on visual memory for faces and line orientations in a short-term task presents a solid approach to studying the precision of memory representation. We found no evidence that more complex objects, such as faces, exhaust visual working memory more quickly than do simpler objects, such as line orientations. Our study underscores the importance of varying memory-to-probe similarity when assessing visual working memory.

\section{Note-Accepted by the editorial board of Editor-Elect Jeremy M. Wolfe.}

\section{AUTHOR NOTE}

The authors contributed equally to this study, which was funded in part by NSF 0733764, NIH 071788, and ARO 46926-LS. We thank Arash Afraz for help with the face stimuli, MiYoung Kwon for help with modeling, and Steve Luck, George Alvarez, Jeremy Wolfe, Kalanit GrillSpector, James Pomerantz, Khena Swallow, Leah Watson, and Galit Yovel for comments and suggestions. Correspondence concerning this article should be sent to any of the authors (e-mail: jiang166@umn.edu, wshim@mit.edu, or tal.makovski@gmail.com).

\section{REFERENCES}

Alvarez, G. A., \& Cavanagh, P. (2004). The capacity of visual shortterm memory is set both by visual information load and by number of objects. Psychological Science, 15, 106-111.

Alvarez, G. A., \& CAVAnagh, P. (2008). Visual short-term memory operates more efficiently on boundary features than on surface features. Perception \& Psychophysics, 70, 346-364.

Attneave, F. (1957). Physical determinants of the judged complexity of shapes. Journal of Experimental Psychology, 53, 221-227.

Attneave, F., \& Arnoult, M. D. (1956). The quantitative study of shape and pattern perception. Psychological Bulletin, 53, 452-471.

Awh, E., Barton, B., \& Vogel, E. K. (2007). Visual working memory represents a fixed number of items regardless of complexity. Psychological Science, 18, 622-628.
BADDELEY, A. [D.] (1986). Working memory. Oxford: Oxford University Press.

Blanz, V., \& Vetter, T. (2003). Face recognition based on fitting a 3D morphable model. IEEE Transactions on Pattern Analysis \& Machine Intelligence, 25, 1063-1074.

Brainard, D. H. (1997). The Psychophysics Toolbox. Spatial Vision, 10, 433-436.

Buttle, H., \& Raymond, J. E. (2003). High familiarity enhances visual change detection for face stimuli. Perception \& Psychophysics, 65, 1296-1306.

Chen, D., ENG, H. Y., \& JiAng, Y. [V.] (2006). Visual working memory for trained and novel polygons. Visual Cognition, 14, 37-54.

Chun, M. M., \& Jiang, Y. [V.] (1998). Contextual cueing: Implicit learning and memory of visual context guides spatial attention. Cognitive Psychology, 36, 28-71.

Cowan, N. (2001). The magical number 4 in short-term memory: A reconsideration of mental storage capacity. Behavioral \& Brain Sciences, 24, 87-185.

Curby, K. M., \& Gauthier, I. (2007). A visual short-term memory advantage for faces. Psychonomic Bulletin \& Review, 14, 620-628.

Delvenne, J.-F., \& BRUYeR, R. (2006). A configural effect in visual short-term memory for features from different parts of an object. Quarterly Journal of Experimental Psychology, 59, 1567-1580.

Duchaine, B., \& NaKayama, K. (2005). Dissociations of face and object recognition in developmental prosopagnosia. Journal of Cognitive Neuroscience, 17, 249-261.

Duncan, J., \& Humphreys, G. W. (1989). Visual search and stimulus similarity. Psychological Review, 96, 433-458.

ENG, H. Y., CHEN, D., \& JIANG, Y. [V.] (2005). Visual working memory for simple and complex visual stimuli. Psychonomic Bulletin \& Review, 12, 1127-1133.

ENG, H. Y., ChEN, D., \& JiANG, Y. [V.] (2006). Effects of familiarity on visual working memory of upright and inverted faces. Journal of Vision, 6, 359A.

Farah, M. J., TAnaka, J. W., \& Drain, H. M. (1995). What causes the face inversion effect? Journal of Experimental Psychology: Human Perception \& Performance, 21, 628-634.

Farah, M. J., Wilson, K. D., Drain, H. M., \& Tanaka, J. R. (1995). The inverted face inversion effect in prosopagnosia: Evidence for mandatory, face-specific perceptual mechanisms. Vision Research, 35, 2089-2093.

GARNER, W. R. (1962). Uncertainty and structure as psychological concepts. Oxford: Wiley.

GARNER, W. R., \& SUTLIFF, D. (1974). The effect of goodness on encoding time in visual pattern discrimination. Perception \& Psychophysics, 16, 426-430.

Grill-Spector, K., \& Malach, R. (2004). The human visual cortex. Annual Review of Neuroscience, 27, 649-677.

Hollingworth, A., Richard, A. M., \& Luck, S. J. (2008). Understanding the function of visual short-term memory: Transsaccadic memory, object correspondence, and gaze correction. Journal of Experimental Psychology: General, 137, 163-181.

IRWIN, D. E., \& ANDREWS, R. V. (1996). Integration and accumulation of information across saccadic eye movements. In T. Inui \& J. L. McClelland (Eds.), Attention and performance XVI: Information integration in perception and communication (pp. 125-155). Cambridge, MA: MIT Press.

JACKSON, M. C., \& RAYMOND, J. E. (2008). Familiarity enhances visual working memory for faces. Journal of Experimental Psychology: Human Perception \& Performance, 34, 556-568.

JiAng, Y. [V.], Chun, M. M., \& Olson, I. R. (2004). Perceptual grouping in change detection. Perception \& Psychophysics, 66, 446-453.

LogIE, R. H. (1995). Visuo-spatial working memory. Hillsdale, NJ: Erlbaum.

LUCK, S. J., \& Vogel, E. K. (1997). The capacity of visual working memory for features and conjunctions. Nature, 390, 279-281.

LUCK, S. J., \& ZHANG, W. (2004). Fixed resolution, slot-like representations in visual working memory [Abstract]. Journal of Vision, 4, 149a.

MAKovsKi, T., \& JiANG, Y. V. (2008a). Indirect assessment of visual working memory for simple and complex objects. Memory \& Cognition, 36, 1132-1143.

MAKovsKi, T., \& JiAng, Y. V. (2008b). Proactive interference from items 
previously stored in visual working memory. Memory \& Cognition, 36, 43-52.

MAKOVSKI, T., SHIM, W. M., \& JIANG, Y. V. (2006). Interference from filled delays on visual change detection. Journal of Vision, 6, 1459-1470.

Moraglia, G. (1989). Display organization and the detection of horizontal line segments. Perception \& Psychophysics, 45, 265-272.

Moscovitch, M., Winocur, G., \& Behrmann, M. (1997). What is special about face recognition? Nineteen experiments on a person with visual object agnosia and dyslexia but normal face recognition. Journal of Cognitive Neuroscience, 9, 555-604.

Palmer, J. (1990). Attentional limits on the perception and memory of visual information. Journal of Experimental Psychology: Human Perception \& Performance, 16, 332-350.

PAsHler, H. (1988). Familiarity and visual change detection. Perception \& Psychophysics, 44, 369-378.

PelLi, D. G. (1997). The VideoToolbox software for visual psychophysics: Transforming numbers into movies. Spatial Vision, 10, 437-442.

Pelli, D. G., Burns, C. W., Farell, B., \& Moore-Page, D. C. (2006). Feature detection and letter identification. Vision Research, 46, 46464674.

PhiLLIPS, W. A. (1974). On the distinction between sensory storage and short-term visual memory. Perception \& Psychophysics, 16, 283-290.

Rouder, J. N. (2001). Absolute identification with simple and complex stimuli. Psychological Science, 12, 318-322.

Rouder, J. N., Morey, R. D., Cowan, N., Zwilling, C. E., Morey, C. C., \& PRATTE, M. S. (2008). An assessment of fixed-capacity models of visual working memory. Proceedings of the National Academy of Sciences, 105, 5975-5979.

Troje, N. F., \& BüLthoff, H. H. (1996). Face recognition under varying poses: The role of texture and shape. Vision Research, 36, 1761-1771.

Wilken, P., \& MA, W. J. (2004). A detection theory account of change detection. Journal of Vision, 4, 1120-1135.
WoLfE, J. M. (1998). What can 1 million trials tell us about visual search? Psychological Science, 9, 33-39.

Wolfe, J. M., Friedman-Hill, S. R., Stewart, M. I., \& O'Connell, K. M. (1992). The role of categorization in visual search for orientation. Journal of Experimental Psychology: Human Perception \& Performance, 18, 34-49.

YIN, R. K. (1969). Looking at upside-down faces. Journal of Experimental Psychology, 81, 141-145.

Young, A. W., Hellawell, D., \& Hay, D. C. (1987). Configurational information in face perception. Perception, 16, 747-759.

ZHANG, W., \& LuCK, S. J. (2008). Discrete fixed-resolution representations in visual working memory. Nature, 453, 233-235.

Zhou, F., Kahana, M. J., \& Sekuler, R. (2004). Short-term episodic memory for visual textures: A roving probe gathers some memory. Psychological Science, 15, 112-118.

\section{NOTES}

1. A bias to choose the more extreme probe can yield above-chance performance for a memory object toward the extreme, whereas the same bias will yield below-chance performance for a memory object toward the middle. In other words, a consistent bias (e.g., of always choosing the more extreme probe) will not produce above-chance performance.

2. We thank George Alvarez for this suggestion.

3. Load 1 is used as an anchor because it is the simplest case, uncomplicated by multiple comparisons. Note that a precise performance match between any two types of stimuli at load 1 is unnecessary. When one type of stimulus produces a psychometric curve that is rightward shifted compared with that of another type, researchers simply need to shift the second curve to match the first, and apply the shift to all memory loads.

4. We thank Steve Luck for raising this important question. 


\section{APPENDIX}

In a recent study, Zhang and Luck (2008) used computational modeling to distinguish the "flexible resolution" account and the "fixed-slot, fixed-resolution" account. Simply stated, the proportion of reporting a test stimulus as being the same as the memory stimulus increases as the memory-to-test similarity increases. If increasing memory load makes memory less precise, then the spread of the error distribution should be broader. Conversely, if some items are held with high precision and others are not at all stored, the error distribution should not get broader, but should be shifted upward by a constant amount corresponding to participants' random guesses for items left out of memory. In other words, the error distribution should maintain its degree of spread, but its tail should asymptote at a value greater than zero.

Using this logic, we fitted a generalized error distribution (also called the exponential power distribution) to our data averaged across all participants, separately for the face and line task (Experiment 1), and separately for each memory load. The probability density function used to model our data (en.wikipedia.org/wiki/Exponential_power_distribution) can be found on Wikipedia, except that we added a third parameter corresponding to the upward shift. The $R^{2}$ of the fit was $.83, .88, .97$, and .89 for loads $1,2,3$, and 4 of the face task, and $.90, .96, .98$, and .99 for loads $1,2,3$, and 4 of the line task

Figure A1A plots the modeled spread of the error distribution at different memory loads, where higher values on the $y$-axis indicated poorer precision (errors spread more widely). Figures A1B and A1C plot the modeled and underlying data at memory load 2 and 4 for faces and lines, respectively. The model was fitted to the averaged data across all participants. The modeling results are broadly, though perhaps not uniquely, consistent with the "fixed-slot, fixed-resolution" view. Specifically, assuming that the number of fixed slots available for lines is greater than four, the increased precision at lower load may be explained by the redundant gain due to duplicated representations in four or more slots. Furthermore, assuming that the number of fixed slots available for faces is two or fewer, the constant precision at all loads may be explained by the fixed-resolution model.

A

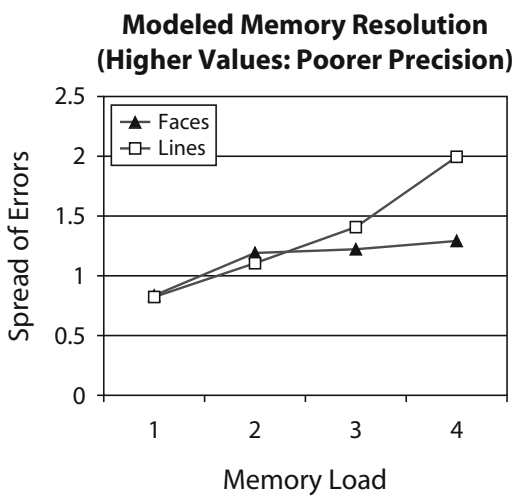

B

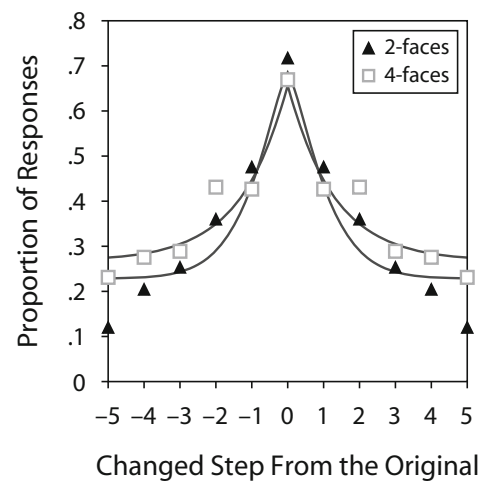

C

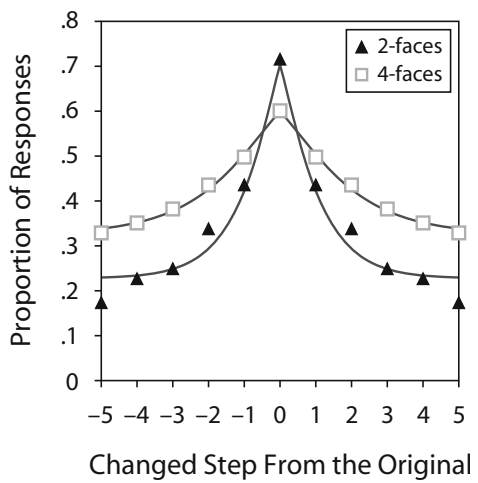

Figure A1. (A) Modeled memory precision (the spread parameter) for lines and faces at different memory loads. Higher numbers on the $y$-axis mean worse precision. Errors spread more widely when line load increased from 1 to 4 , but was relatively constant for faces. (B) Model fit for Face 2 and Face 4. (C) Model fit for Line 2 and Line 4.

There are several caveats in this modeling attempt that may weaken the conclusions one can reach. First, the fit for individual participants was worse ( $R^{2}$ ranging from .62 to .98) than the fit for the averaged data. Second, we used a two-alternative forced choice rather than a free choice of all 11 possible stimuli, so the 0 -step change (the choice that matched the memory object) was overrepresented in the data. This may have contributed to the "peakier" envelop than Gaussian functions in the error distribution. Finally, the 11 possible stimuli did not cover the entire stimulus space (e.g., we only sampled $20^{\circ}$ to $70^{\circ}$ in orientation space and only sampled the average face to Face A). Some of the stimuli (e.g., the $90 \%$ Face A) could only be tested with a probe that was less extreme than the original. These factors may affect the underlying error distribution, reducing the confidence of the data fitting.

(Manuscript received January 14, 2008;

revision accepted for publication June 27, 2008.) 